\title{
Liver carcinogenesis: diagnostic and clinical aspects of preneoplastic nodules
}

\author{
Mauro Borzio', Fabio Paladino², Giampiero Francica ${ }^{3}$ \\ 'U.O. Gastroenterologia ed Endoscopia Digestiva, ASST Melegnano-Martesana, Melegnano, Vizzolo Predabissi 20070, MI, Italy. \\ U.O. Chirurgia, ASST Melegnano-Martesana, Melegnano, Vizzolo Predabissi 20070, MI, Italy. \\ ${ }^{3}$ U.O. Ecografia ed Econterventistica, Pineta Grande Hospital, Castel Volturno 81030, CE, Italy.
}

Correspondence to: Dr. Mauro Borzio, Ospedale Uboldo, Cernusco sul Naviglio 20063, MI, Italy. E-mail: mauro.borzio@gmail.com

How to cite this article: Borzio M, Paladino F, Francica G. Liver carcinogenesis: diagnostic and clinical aspects of preneoplastic nodules. Hepatoma Res 2019;5:15. http://dx.doi.org/10.20517/2394-5079.2019.11

Received: 12 Feb 2019 First Decision: 18 Mar 2019 Revised: 12 Apr 2019 Accepted: 18 Apr 2019 Published: 14 May 2019

Science Editor: Jin-Lin Hou Copy Editor: Cai-Hong Wang Production Editor: Huan-Liang Wu

\begin{abstract}
In multistep hepatocarcinogenesis, sizable lesions can precede the development of hepatocellular carcinoma (HCC). These lesions are currently classified as low grade (LG)- and high grade (HG)-dysplastic nodules. Following international guidelines recommending the surveillance of cirrhotic patients, a growing number of $1-2 \mathrm{~cm}$ hepatocellular nodules are recognized including early hepatocellular carcinoma (eHCC) and DN the latter accounting for as many as $70 \%$ of nodules $<1 \mathrm{~cm}$. HG-DN are currently considered the most advanced HCC precursors. The histological diagnosis of low-grade dysplastic nodule (LG-DN), high- grade dysplastic nodule (HG-DN) and eHCC in small liver biopsies requires a comprehensive stepwise morphological and immunocytochemical approach. By imaging the differential diagnosis among these lesions is a challenge. According to vascular enhancement at dynamic computed tomography (CT) or magnetic resonance imaging (MRI) these precursors are classified as hypo-vascular/ indeterminate nodules even though distinction between LG-DN and HG-DN is almost impossible. The introduction of gadoexetic acid-enhanced MRI has represented an extremely important advance in this field allowing a better differentiation of dysplastic lesions from eHCC and progressed HCC. Additional MRI features as diffusion-weighted imaging further improved diagnostic accuracy of imaging. According to Liver Imaging Reporting and Data System (LIRADS), either CT/MRI or Contrast-Enhanced Ultrasound LI-RADS, the dysplastic lesions should be categorized as LR-3 or LR-4. Natural history of these lesions confirmed that HCC can develop from HG-DN but which nodule and when it will undergo malignant transformation is not predictable. The search and validation of radiological and tissue markers able to select lesions more prone to HCC development, is currently underway. Whether and how HG-DN should be ablated or closely followed up is currently debated.
\end{abstract}

Keywords: Low-grade dysplastic nodule, high-grade dysplastic nodule, early hepatocellular carcinoma, progressed hepatocellular carcinoma, dynamic imaging, gadoexetic acid-enhanced resonance imaging, hepatobiliary phase

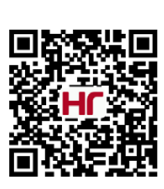




\section{INTRODUCTION}

It has long been known that, like in others human carginogenetic models as colo-rectal cancer, in hepatocellular multistep carcinogenesis precancerous lesions both micro- and macroscopic precede the development of hepatocellular carcinoma (HCC ${ }^{[1,2]}$. Chronic hepatitis and cirrhosis represent the natural background in which precancerous lesions grow through stepwise sequence. These lesions have been described many years ago and include microscopic dysplastic foci (DF) and sizable dysplastic nodules (DN). The latter are further categorized as low-grade (LG-DN) and high-grade dysplastic nodules (HG-DN) with specific and distinctive morphologic characteristics and different carcinogenetic propensity ${ }^{[3]}$. Representation of multistep hepatic carcinogenesis is schematized in [Figure 1]. Given the low attitude to perform liver biopsy in cirrhotic patients, microscopic preneoplastic lesions have been losing interest in clinical practice. Hence, the present review will focus specifically on preneoplastic nodules which can be routinely detected by radiologic techniques and monitored over time for their potential neoplastic transition. Histologic classification, histopatologic features, radiologic diagnostic criteria, natural history and treatment of these lesion will be discussed.

In cirrhosis, application of regular ultrasound surveillance resulted in the discovery of an increasing number of small nodules $[<2 \mathrm{~cm}$ according to the definition of small lesion by International Working Party $(\mathrm{IWP})]^{[4]}$. Small nodules usually include definite hepatocellular carcinoma (HCC), either early hepatocellular carcinoma (eHCC) and very-early hepatocellular carcinoma (veHCC) or progressed hepatocellular carcinoma (pHCC) and the large majority of pre-neoplastic nodules ${ }^{[5-9]}$. Other small lesions as metastases or mesenchymal neoplasms are much less frequently detected during surveillance.

Distinction of pre-neoplastic nodules from well-established HCC is mandatory for decision making process, particularly in transplant or surgical setting. To decide whether a given nodule is malignant, premalignant or benign is therefore of paramount importance demanding a great radiologic, morphologic and clinical expertise. The following key points should help clinicians to get oriented in the management of such small nodules:

1. Precancerous nodules have a maximum diameter seldom exceeding $2 \mathrm{~cm}$ and are typically detected in cirrhosis/chronic hepatitis.

2. Their prevalence is relevant ranging from $60 \%$ to $70 \%$ among nodules $<1 \mathrm{~cm}$ and from $20 \%$ to $30 \%$ among nodules $>1<2 \mathrm{~cm}$.

3. They can be intercepted as single or multiple lesions and often concomitant with already established HCC.

4. HG-DN account for $30 \%$ of all precancerous nodules and are considered true precancerous lesions whereas LG-DN have a trivial neoplastic risk comparable to that of large regenerative nodules.

5. Transforming risk of HG-DN is largely unpredictable at baseline.

\section{HISTOPATOLOGICAL CLASSIFICATION AND FEATURES OF DYSPLASTIC NODULES}

\section{Nomenclature}

Definition and nomenclature of precancerous nodules was firstly proposed by the International Working Party in $1995^{[4]}$ and further updated in an east-western consensus of pathologists in $2008^{[10]}$.

Non-malignant hepatocellular nodules include large regenerative nodules (LRN) and DN. The latter are subclassified as LG-DN and HG-DN. However, LG-DN share a number of features to non-neoplastic LRN and therefore, the distinction between these two lesions is particularly difficult and unpractical. Hence, the panel of experts recommended not to separate LG-DN from LRN and classify as LG-DN any nodule that cannot be classified as HG-DN ${ }^{[4]}$. Their size ranges from few millimetres to $2 \mathrm{~cm}$ rarely exceeding $3 \mathrm{~cm}$.

\section{Histologic differential diagnosis}

Histologic differentiation of LG-DN, HG-DN and eHCC is challenging, particularly when pathologists have to deal with small samples obtained by fine needle biopsy. It has been repeatedly stressed that histology is 


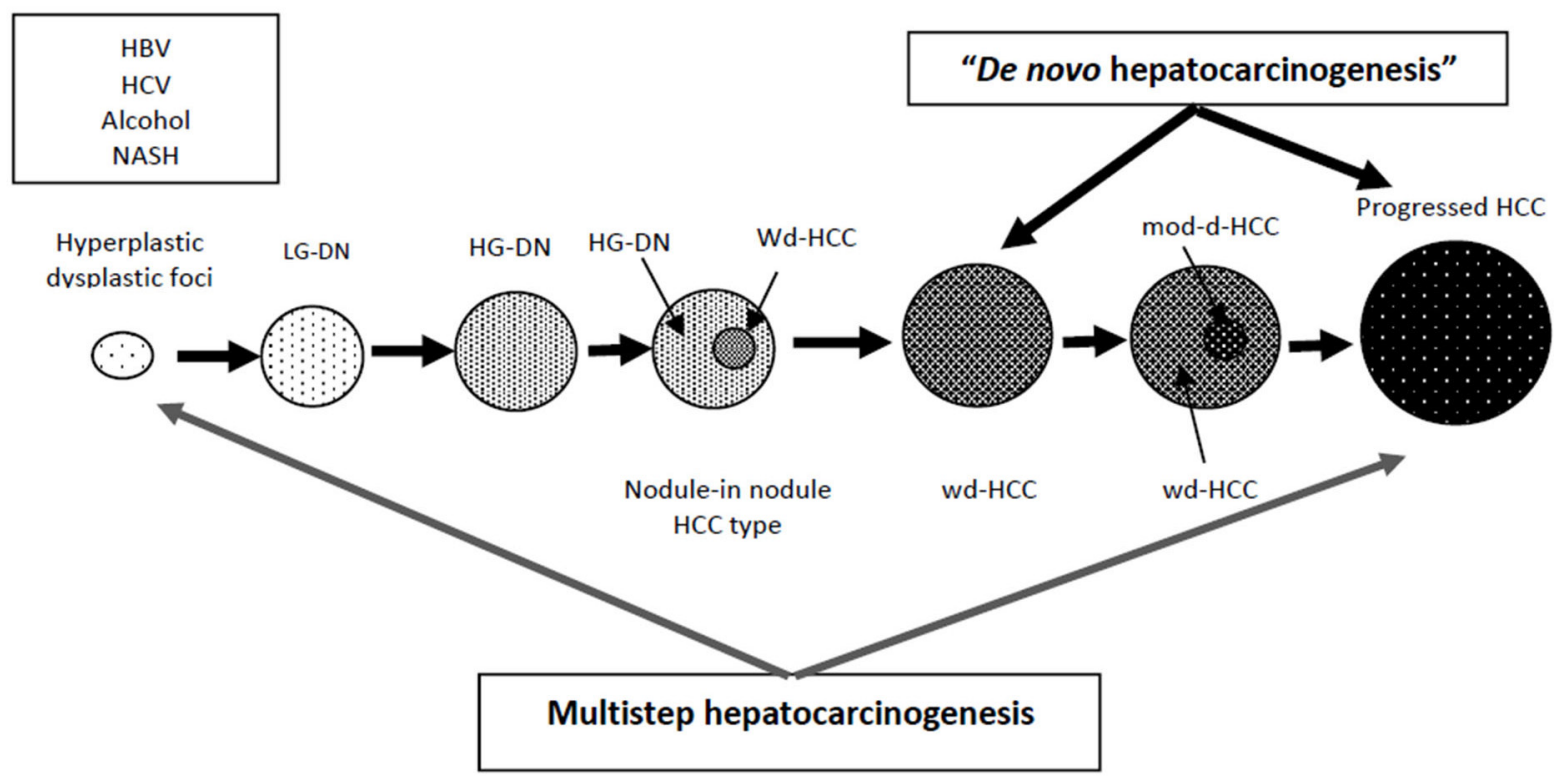

Figure 1. Schematic illustration of lesions occurring in hepatocarcinogenesis. Two models of human hepatocarcinogenesis. HCC: hepatocellular carcinoma; DN: dysplastic nodules; HG-DN: high-grade dysplastic nodules; HG-DN: low-grade dysplastic nodules

mandatory, and that comparison between intra nodular and extra nodular tissue is of great importance. Cytology should be avoided since it does not allow to appreciate architectural changes useful for differential diagnosis. First diagnostic step is to evaluate whether or not the sample is adequate. Schematically, adequacy can be checked by evaluating the overall nodularity at low magnification [Figure 2] by comparing intraand extranodular samples. In brief, when both intra- and extra-lesional samples show overlapping cirrhosislike features, without a major nodule in the background, the sample should not be considered adequate and biopsy should be repeated shortly after. This is another reason why cytology is inadequate to approach this issue.

Small liver cell dysplasia (SLCD) is a key feature of HG-DN. It refers to areas of increased cell density (more than twice that of the surrounding tissue) and plate thickening, simulating a well-differentiated HCC. Formation of pseudoglands/acini along with SLCD are features helping to distinguish HG-DN from LG-DN. These features are also present in eHCC even though much more pronounced.

Unpaired arteries, so called because they are not coupled with bile ducts, is another distinctive feature of liver carcinogenesis and represent neoangiogenesis which is the morphologic counterpart of arterial contrast enhancement on imaging. Similarly, sinusoidal capillarization which can be highlighted by CD34 immunostaining is minimal in cirrhosis and increases from LG- to HG-DN with the highest levels in HCC.

Reticulin framework is another feature useful to distinguish benign from malignant nodules since it is usually well preserved in both regenerative and dysplastic nodules whereas it is decreased or lost in advanced HCC; however, in early well-differentiated HCC, the reticulin framework may be retained or only slightly decreased.

Portal tracts are retained in HG-DN and can be retained in eHCC as well and their presence does not help differential diagnosis.

Stromal invasion is the key feature of definitive malignant transition separating eHCC from HG-DN. Stromal invasion has to be looked for in portal tracts but, unfortunately, it is not always detectable, especially on samples obtained by fine needle biopsy. For iconography please refer to recent review by Roncalli et al. ${ }^{[11]}$. 


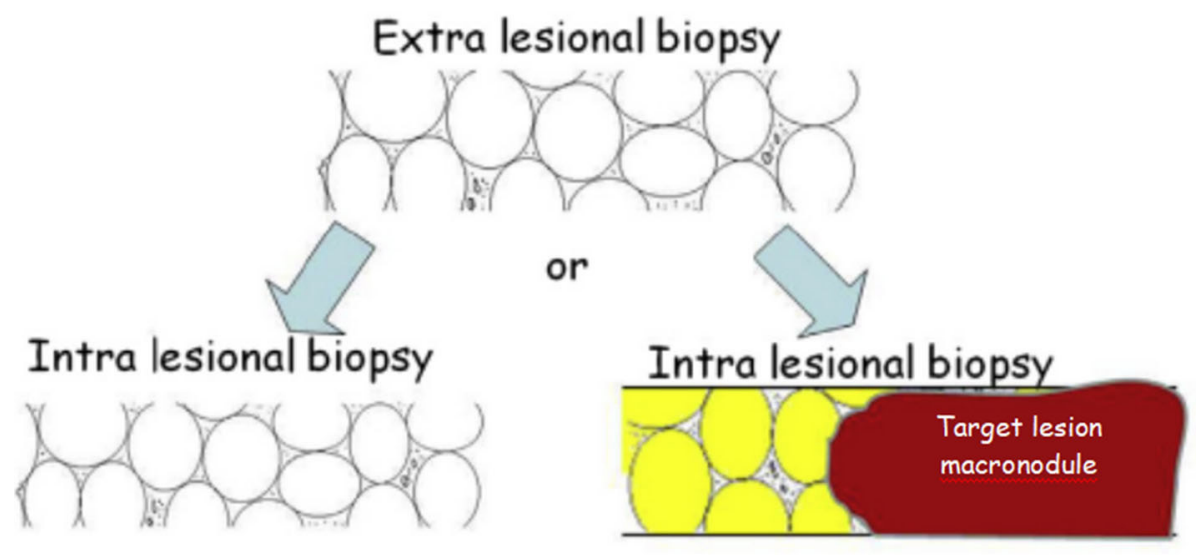

Inadequate

Adequate

Figure 2. Schematic illustration of adequacy judgement of liver biopsy for small nodules in cirrhosis. Intra- and extra-lesion samples are mandatory. Schematically, adequacy can be checked by evaluating the overall nodularity at low magnification by comparing intra- and extranodular biopsy samples. When both intra- and extra-lesional samples show overlapping cirrhosis-like features, without a major nodule in the background, the sample should not be considered adequate and biopsy should be repeated shortly after

The immunostaining of tissue biomarkers is a promising tool in the armamentarium of pathologists for improving the accuracy of the differential diagnosis between benign and premalignant/malignant nodules [Table 1]. In the last two decades, translation of data from gene expression profiles to clinical practice has been focused on the search of serum and tissue markers involved in hepatocarcinogenesis and useful for diagnostic purposes. Most of these markers can be tested on paraffin embedded, hematoxilin-eosin stained tissue samples making this diagnostic technique suitable for employment in clinical practice even on fine needle liver biopsies. Among them alpha-fetoprotein and des- $\gamma$-carboxy phrotrombin are serum markers of HCC but are not sensitive enough for $\mathrm{eHCC}^{[12]}$. Other tissue markers as heat shock protein 70 (HSP70), glutamine synthetase (GS), glypican 3 (GPC3), CD34, p53, proliferating cell nuclear antigen (PCNA) and Ki67 have been tested in lesions at different neoplastic evolution in the multistep process leading to mature HCC $^{[12]}$. A panel of three biomarkers: HSP70, GS and GPC3 has been applied to differentiate non-malignant nodules (either LG or HG-DN) from HCC and a positivity for any two or three markers detected malignancy with a sensitivity of $72 \%$ and specificity of $100 \%$. Sensitivity dropped to $50 \%$ when the panel was applied to tissue obtained by fine needle biopsy samples whereas specificity remained absolute ${ }^{[13]}$. These findings were validated by other authors ${ }^{[14]}$. More recently it has been demonstrated that the addition of clathrin heavy chain (CHC) to the above-mentioned panel of biomarkers increased the diagnostic accuracy for small HCCs from $76.9 \%$ to $84.3 \%$, with an important gain in sensitivity (from $46.8 \%$ to $63.8 \%{ }^{[15]}$. Currently, the use of three biomarkers panel with CD34 is endorsed by AASLD-EASL guidelines as a diagnostic instrument for diagnosis of HCC $^{[16,17]}$. An algorithmic workflow based on sample adeguacy and histopatological features including biomarkers immunostaining useful for characterization of small nodules in cirrhosis has been recently proposed by Roncalli et al. ${ }^{[11]}$.

\section{The role of biopsy}

International guidelines recommend performing a biopsy whenever a nodule results atypical or indeterminate at conventional dynamic imaging (see below) ${ }^{[16-20]}$. If a biopsy should be done, a 18-20 gauge cutting needle should be employed and intra- and extra-lesion sample should be obtained. In the setting of small nodules in cirrhosis however, the role of biopsy is hampered by the risk of false negative results due to sampling error. Smaller the nodule higher the risk of sampling error. The rate of false negative sample is quite high and a successful result achievable in no more than two-third of patients ${ }^{[8]}$. In the remaining one-third of cases a second biopsy within a short interval is recommended. It is important to note that, contrarily to 
Table 1. Morphologic features and diagnostic tools for differential diagnosis between dysplastic nodules and early HCC

\begin{tabular}{|c|c|c|c|c|}
\hline & LG-DN & HG-DN & eHCC & $\begin{array}{l}\text { Diagnostic value between } \\
\text { HG-DN and eHCC }\end{array}$ \\
\hline \multicolumn{5}{|l|}{ Elementary morphologic feature } \\
\hline \multicolumn{5}{|l|}{ Parenchimal changes, cytologic alterations } \\
\hline SCC & - & + & + & Low \\
\hline LCC & \pm & \pm & - & Low \\
\hline Clone like & \pm & + & + & Low \\
\hline \multicolumn{5}{|l|}{ Architectural changes } \\
\hline Cell density & \pm & + & + & Low \\
\hline Pseudoglands/acini & - & \pm & + & Medium \\
\hline \multicolumn{5}{|l|}{ Non-parenchymal changes } \\
\hline Portal tracts & + & + & \pm & Low \\
\hline Reticulin framework & + & + & \pm & Medium* \\
\hline Umpaired arteries or sinusoidal capillarization (CD34) & \pm & \pm & + & Low \\
\hline \multicolumn{5}{|l|}{ Diagnostic tools } \\
\hline Stromal invasion/loss of ductular reaction (K7/19) & - & - & \pm & High \\
\hline $\begin{array}{l}\text { HCC biomarkers expression (at least } 2 \text { markers among HSP70, } \\
\text { GPC3, GS, CHC) }\end{array}$ & - & - & -+ & High \\
\hline Nodule-in-nodule & - & - & \pm & High \\
\hline
\end{tabular}

*If frankly decreased or lost, the discriminatory value of reticulin framework is high. -: absent; \pm : may be present but not necessarily detectable in biopsy; +: present and usually detectable in biopsy. CHC: clathrin heavy chain; GPC3: glypican 3; HSP70: heat shock protein 70; LCC: large cell change; SSC: small cell change; LG-DN: low-grade dysplastic nodule; HG-DN: high-grade dysplastic nodule; eHCC: early hepatocellular carcinoma

what can be expected, a second biopsy has the same probability of success as the first. Thus, repeated biopsy would finally lead to a successful diagnostic yield in more than $90 \%$ of cases ${ }^{[8,21]}$. However, this invasive strategy is seldom adopted in clinical practice mainly for the awareness that a priori probability that such indeterminate $1-2 \mathrm{~cm}$ nodules are malignant is $l_{0} w^{[8,9,22,23]}$ and that $2 \mathrm{~cm}$ is the size threshold to achieve the best result by percutaneous ablation. Based on these assumptions, a wait and see strategy with the adoption of a strict monitoring of the nodule by dynamic imaging is often preferred (see below). Nodule location and coagulative disorders are additional features making biopsy difficult or impossible. Lastly, the potential and theoretical risk of tumour seeding, should be considered even though, this risk, ranging between $1 \%$ and $2.7 \%$, seems to balance favourably with the risk of inappropriate or delayed treatment ${ }^{[24-26]}$.

\section{RADIOLOGIC CRITERIA FOR DIAGNOSIS AND CHARACTERIZATION OF PRENEOPLASTIC NODULES IN CIRRHOSIS DYNAMIC IMAGING CT AND MRI}

To date, the differential diagnosis between eHCC and dysplastic nodules still remains a radiologic challenge. Premalignant nodules are usually detected by standard US as hypo- or, less frequently, as hyper-echoic nodules completely indistinguishable from well-established HCC. Thus, standard US is inadequate for differential diagnosis and other imaging tools are needed. The physiopathologic basis for the diagnosis and characterization of small hepatic nodules by imaging rests on characteristics of their vascular supply. It is well established that during cirrhosis-related oncogenesis, progressive loss of normal portal vascular supply in favour of increase arterial one, the so called neoangiogenesis, occurs. This nodular arterialization becomes progressively more evident by transition from regenerative to dysplastic and neoplastic nodules reaching the full expression in high-differentiated HCCs. Dynamic CT, magnetic resonance imaging (MRI) and ContrastEnhanced Ultrasound (CEUS) are the contrast-enhanced imaging to investigate the vascular pattern of nodules detected under surveillance in cirrhosis. A recent meta-analysis including several comparative studies confirmed that MRI is more accurate than dynamic CT for detection and characterization of small lesions and therefore, it should be preferred as a first line panoramic imaging ${ }^{[27]}$. In addition, a comparative 13-years meta-analysis ${ }^{[28]}$ showed that CEUS had a sensitivity and PPV close to that of MRI with gadoexetic contrast media. 
At imaging using the so-called extracellular contrast media, mature HCC shows a typical vascular pattern characterized by homogeneous and intense contrast uptake in the arterial phase known as "wash in" followed by a progressive washout of contrast in venous or late phases. Opposite, regenerative nodules, being a simple hypertrophic inflammatory growth of normal hepatocytes, maintain a normal vascular supply and share the same contrast uptake as the surrounding cirrhotic parenchyma during arterial and venous phases. Dysplastic nodules, either LG-DN or HG-DN lack a well- developed neoangiogenesis and maintain normal or partially normal portal inflow appearing as non-enhancing or partially enhancing nodules. Conventional dynamic imaging "per se" cannot differentiate LG-DN from HG-DN. Diagnostic difficulties are also represented by those incipient eHCCs with still poorly developed neoangiogenesis which usually appear as non-enhancing lesions (the so-called hypovascular HCC) and, therefore, hardly distinguishable from HG-DNs.

According to all the international guidelines ${ }^{[16-20]}$ arterial wash-in and late washout, when present, are sufficient for the diagnosis of HCC and histologic confirmation is not necessary even for small lesions. This paradigm served to design the non-invasive diagnostic algorithm for nodules detected in cirrhotic patients under surveillance proposed by AASL-EASL-ERTOC guidelines ${ }^{[16-17]}$. According to this algorithm, new lesions $<1 \mathrm{~cm}$ should be strictly monitored without biopsy. Nodules $>1 \mathrm{~cm}$ showing definite hallmarks of malignancy by a single enhancing imaging do not require confirmation by a second one and diagnosis of HCC is accepted. In case of equivocal/inconclusive results by the first imaging, a second one should be sequentially performed. If uncertainty still persists after two dynamic techniques, lesion should be categorized as indeterminate or atypical and biopsy is recommended ${ }^{[16,29]}$.

This sequential strategy demonstrated high specificity and almost absolute PPV for diagnosis of HCC and, in clinical practice, proved to be cost-effective and useful for minimizing the number of futile biopsies ${ }^{[9,22]}$.

Although specificity and positive predictive value of radiologic hallmarks of malignancy (wash in and late washout) are close to $100 \%$, their sensitivity in small lesions is suboptimal $(71 \%)^{[30,31]}$. According to vascular-based imaging modalities one-third of small nodules discovered under surveillance remain indeed indeterminate or atypical even by dynamic MRI. Indeterminate/atypical lesions include approximately 30\% of hypovascular eHCCs ${ }^{[7,22,32]}$ and the large majority of pre-neoplastic lesions (the one-third rule: one-third of small lesions are indeterminate and one-third of them are HCCs).

Premalignant nodules are usually detected by standard US as hypo- or, less frequently, as hyper-echoic small nodules. Hence, standard US cannot be used for differential diagnosis being dysplastic nodules completely indistinguishable from well-established HCC. On dynamic imaging these nodules appear as non- or weakly enhancing lesions on arterial phase and hypo/iso-enhancing on venous/delayed phases (hypovascular nodules). Therefore, they fall into the group of indeterminate/atypical lesions and, as recommended by AASLD/EASL guidelines, they should require histologic diagnosis. In this setting however, the role of biopsy is still debated (see above). The differentiation between DN and eHCC is challenging on conventional dynamic imaging. The recent introduction of MRI hepato-specific post-vascular contrast media has represented a relevant diagnostic advance in the characterization of small nodules in cirrhosis ${ }^{[33,34]}$. Gadolinium-chelate agents as gadolinium ethoxybenzyl diethylenetiamine pentaacetic acid (Gd EOB DTPA, Primovist ${ }^{\top}$ ) or gadobenate dimeglumine (Gd-BOPTA, MultiHance ${ }^{\odot}$ ) enter the hepatocyte by the organic anion transporter polypeptide 1 (OATP1) and are excreted in the bile canaliculi by $\mathrm{MRP}_{2,3,4}$ protein transporters. These agents make possible to achieve information not only on the vascular profile of a given lesion (dynamic phase) but also on specific hepatocyte functions in the so-called hepatobiliary phase (HE phase). During carcinogenetic process, proteins responsible of uptake and excretion of gadolinium-chelates are progressively lost and this derangement may precede changes on vascular profile ${ }^{[35]}$. Most eHCCs and veHCCs, are in fact no longer able to incorporate gadolinium-chelates and appear hypo-intense on HE phase. Opposite, LG-DN and most HG-DN maintain still preserved uptake function and consequently 
appear as iso- or even hyper-intense nodules. These contrast agents are therefore potentially able to distinguish differently progressed lesions ${ }^{[35]}$. Although the full potential of liver-specific-contrast media needs to be fully assessed in prospective studies, the use of these agents is now considered mandatory in this setting since they provide better diagnostic accuracy than the vascular contrast media ${ }^{[36]}$. Recent data on histologically well characterized small atypical nodules in cirrhosis, confirmed the high diagnostic accuracy of Gd EOB DTPA MRI to correctly distinguish eHCC from premalignant nodules. Ninety-seven percent of iso/hypovascular nodules were correctly classified as premalignant or malignant in HE-phase with a specificity of $100 \%$ for malignancy when hypointensity in HP phase was coupled with hypervascularity in arterial phase ${ }^{[32]}$. These data are well in keeping with those of Choi et al. ${ }^{[37]}$. In a recent prospective study ${ }^{[38]}$ carried out on 111 small nodules defined as atypical at CEUS or dynamic CT and histologically classified as eHCCs or dysplastic, either LG-DN and HG-DN, emerged that HE-phase hypointensity by itself using Gd-EOB-DTPA was the strongest predictor of malignancy superior to vascular-based hallmarks and T2w behaviour. All hypointense nodules at HE phase were malignant/premalignant (overt HCC or eHCC/HGDN) and hypointensity captured 45/51 malignant nodules either hyper- or hypovascular, with a sensitivity of $88 \%$ and specificity of $97 \%$ reaching $100 \%$ when associated with arterial enhancement. In this study, all benign nodules (large regenerative or LG-DN) displayed iso-hyperintensity at HE-phase. However, it could be outlined that a very low rate (roughly $3 \%$ ) of well-differentiated hepatocellular carcinomas (wdHCCs), are hyper vascularized at arterial phase but are iso-hyperintense on HE-phase mainly because they retain still functioning hepatocytes ${ }^{[39-43]}$. It is wise to consider nodules with arterial enhancement but hyperintense in HE as highly suspicious of incipient malignant transition.

The differential diagnosis between HG-DN and eHCC still remains difficult even by Gd-EOB-DTPA MRI since HG-DN show variable behaviour on HE-phase being frequently hypointense like eHCC ${ }^{[38,44-48]}$. Indeed, the specificity of hypointensity on HE-phase as a unique hallmark of malignancy, is suboptimal ranging from $33 \%$ to $97 \%{ }^{[38,49]}$. It has been recently outlined that coupling Gd-EOB-DTPA MRI with diffusionweighted imaging (DWI) may result in increased accuracy for diagnosis of overt HCC and also for the differential diagnosis of eHCC and HG-DN ${ }^{[4,45]}$. DWI is based on the simple assumption that water diffusion in the extracellular compartment is influenced by cellular density which, in turn, reduces the width of interstitial spaces and water diffusion ${ }^{[50,51]}$. Water diffusion can be quantified by a mathematical index called apparent diffusion coefficient (ADC); low ADC values mean low diffusivity, namely hypercellularity and, as previously stated, hypercellularity progressively increases from HG-DN, to eHCC reaching maximum expression in progressed HCC. Unfortunately, DWI as a unique tool for assessing hepatic lesions is inaccurate due to the considerable overlap between benign and malignant lesions and normal liver tissue ${ }^{[52,53]}$. In addition, DWI images are highly influenced by artefacts of liver motion due to respiration and artefacts in the left lobe derived from the heart beating ${ }^{[54,55]}$. Therefore, DWI should be used in conjunction to the other conventional imaging features. Renzulli et al.$^{[56]}$ reported data from a prospective study evaluating the imaging criteria of HCC, early HCC and HG-DNs using gadoxetic acid-enhanced liver MRI in 228 patients prospectively enrolled with 480 small nodules detected under surveillance for cirrhosis. Using three MRI findings: HE-phase hypointensity, arterial hyperintensity and diffusion-weighted imaging (DWI) the authors designed an algorithm which yielded an overall sensitivity of $96.6 \%$ and a specificity of $92.7 \%$ and a very high sensitivity (94.7\%) and specificity (99.3\%) in classifying HG-DN. Although some technical aspects need to be fully standardized (type of MRI machine, breath old method, $b$ value for DWI), gadoxetic acid-enhanced MRI including DWI analysis as part of a diagnostic algorithm, may represent a promising approach to better defining those small lesions still atypical at vascular and HE phases being eHCC hyperintense and HG-DN isointense al DWI. DWI behaviour is currently part of ancillary features for liver nodules characterization in Liver Imaging Reporting and Data System (LI-RADS) v17 system (see above) ${ }^{[57]}$.

These advances on MRI prompted JHS and APASL guidelines to incorporate gadoxetic acid-enhanced MRI for definition of atypical nodules before performing biopsy ${ }^{[18,19]}$. Conversely, the late version of guidelines from the Western world ${ }^{[58,59]}$, still endorse a diagnostic algorithm for HCC that have remained the same for 
at least 16 years not giving due relevance to the above discussed imaging innovation. Furthermore, western guidelines erroneously consider CT as equivalent to MRI in diagnostic accuracy even though recent metaanalyses ${ }^{[60-61]}$, one of which conducted by the same authors who contributed to the last version of AASLD guidelines, confirmed, the superiority of the latter. Other hepato-specific contrast media as Sonazoid for CEUS (s-CEUS) or Super paramagnetic iron oxide agents for MRI (SPIO-MRI) showed promising results but they are not available worldwide and the experience with these compounds is limited to Eastern countries ${ }^{[18,19]}$. Sonazoid (GE Healthcare, Waukesha, WI, USA), is a second-generation contrast agent available in Japan, South Korea, and Norway (August 2015). The microbubbles of this agent are captured in the liver parenchyma by reticuloendothelial (Kupffer) cells and, therefore, s-CEUS provides information in both haemodynamic-phase and accumulated-phase images. Because malignant tumours contain few or no Kuppfer cells, they appear as perfusion defects in late phase of s-CEUS. Conversely, non-neoplastic nodules with preserved or only minimally reduced reticuloendothelial system lack wash-out in the latephase. Sonazoid proved to be particularly accurate for characterization of small lesions in cirrhosis. In a literature review covering 10 years experience with Sonazoid ${ }^{[62]}$, s-CEUS emerged as accurate as gadoxetic acid-enhanced MRI in differentiating benign nodules from wdHCC. Furthermore, s-CEUS provides a better characterization of some small lesions at contrast-enhanced CT or MRI. Takahashi et al. ${ }^{[63]}$ in a study comparing s-CEUS and gadoxetic acid-enhanced MRI on characterization of small $<3 \mathrm{~cm}$ lesions in cirrhosis reported that $27 \%$ of indeterminate hypo-vascular lesions at MRI turned out to be hyper-enhancing at s-CEUS and were histologically diagnosed as HCC. For these reasons s-CEUS is currently included by Japanese and APASL guidelines in the diagnostic algorithm for indeterminate lesions discovered in cirrhosis during surveillance.

\section{LI-RADS CRITERIA}

In 2011 the American College of Radiology issued a new system of liver imaging reporting (LI-RADS) aimed at providing a precisely defined terminology for interpreting and reporting contrast-enhancing CT and MRI examination of nodules detected in cirrhosis ${ }^{[64]}$. This system also included guidance for the interpretation of data obtained by MRI with hepato-specific agents. According to LI-RADS, lesions detected at dynamic CT or MRI in patients at high risk of having HCC (cirrhotic patients under surveillance), are categorized as definitively benign (LR-1), probably benign (LR-2), intermediate probability of being HCC (LR-3), probably HCC (LR-4) and definitively HCC (LR-5). This system would provide more guidance to clinicians to adopt proper clinical decisions such as accelerated follow-up, biopsy or even treatment of small lesions in cirrhosis. According to criteria proposed by LI-RADS the great majority of dysplastic nodules are categorized as LR-3 and LR-4. LR-3 lesions should be strictly monitored and LR-4 lesions require biopsy ${ }^{[64]}$.

LI-RADS system was strongly encouraged by the US regulatory agency for liver transplantation (UNOS) to avoid or minimize futile transplants for false positive cases. Indeed, LI-RADS criteria proposed by LIRADS v2014 and by the updated 2017 version (v2017 ${ }^{[57]}$ for diagnosis of HCC are more restrictive than those proposed by AASLD-EASL and do not accept the diagnosis of definitive-HCC (LR-5) for lesions sized 1-2 cm even in presence of wash in and washout. In these cases a 50\% growth in at least 6 months is needed for definite classification as LR-5. These stringent criteria would limit de facto the early diagnosis of HCC in the group of 1-2 cm nodules. These limits were emphasized by a prospective study comparing LI-RADS classification with AASLD criteria for HCC on 133 small $(<2 \mathrm{~cm})$ newly detected nodules in cirrhosis studied by $\mathrm{MRI}^{[65]}$. In this study 21 histologically proven eHCCs were erroneously subcategorized as LR-4 according to LI-RADS criteria even though they were hyper-enhancing at arterial phase with late wash-out. In addition, 29 small HCCs fell into LR-3 category making the overall sensitivity of LI-RADS for HCC 43\%, relevantly lower than the sensitivity of AASLD criteria (58.6\%). Similar results were obtained by Ronot et al. ${ }^{[6]}$ in a prospective study on 595 nodules $<3 \mathrm{~cm}$ in cirrhosis, where specificity of LI-RADS criteria for diagnosis of HCC relevantly increased and became better than AASLD criteria when LR-4 and LR-5 categories were considered in combination. On the other hands and more important, in the study by Darnell et al. ${ }^{[65]}$ 
none of the non-malignant hepatocellular nodules fell into LR5 category making the LI-RADS specificity for HCC diagnosis close to $100 \%$. The high specificity of LI-RADS criteria was further emphasized in a recent systematic review in which data from 2,760 patients were analysed ${ }^{[67]}$. The authors found that only $3 \%$ of observations categorized as LR5 were non-malignant giving a $97 \%$ overall specificity of LI-RADS for malignancy. To overcome the low sensitivity of LI-RADS, the most recent version (v2018) ${ }^{[68]}$ provides updated criteria for small (10-19 mm) LR-5 observations and a simplified definition for threshold growth. In this version, in order to align LI-RADS criteria with AASLD/EASL criteria of HCC and increase simplicity, a 10-19 mm nodule with arterial phase hyperenhancement and non-peripheral "washout" is definitely categorized as LR-5.

According to LI-RADS v2018, hypointensity in the HE-phase and DWI hyperintensity are considered as ancillary features but they should not allow upgrading a suspected malignant lesion (LR-4) to LR- ${ }^{[66]}$. In order to significantly increase the diagnostic efficacy of the MRI LI-RADS criteria it has been suggested that they should be modified (mLI-RADS) by incorporating hypointensity in the HE-phase and hyperintensity at diffusion restriction among the major MRI features of malignancy ${ }^{[68]}$.

A further progress in the diagnostic accuracy small HCCs comes from the recently released ContrastEnhanced Ultrasound LI-RADS (CEUS LI-RADS v2017) which provides a refined definition of the typical CEUS pattern of $\mathrm{HCC}^{[69]}$. According to CEUS LI-RADS criteria, a nodule showing a rapid arterial enhancement and a delayed wash out (> $60 \mathrm{~s}$ ) can be classified as definite HCC (LR-5) regardless its size. These criteria were recently validated in a series of 1086 well-defined lesions detected in cirrhosis and studied by CEUS. Applying CEUS LI-RADS criteria $58.5 \%$ of HCCs were correctly classified as LR- 5 with a PPV of $98.5 \%$ and only $3 \%$ of non-malignant nodules were erroneously classified as HCC ${ }^{[70]}$. Thus, LR-5 CEUS is an extremely reliable criteria for HCC, given its excellent PPV, without misdiagnosis for other malignancies. Using CEUS LI-RADS criteria most of LG and HG-DN were classified al LR-3 and LR-4 ${ }^{[70]}$. These results are extremely important and support the use of CEUS in clinical practice not only for the definite diagnosis of HCC when other imaging is inconclusive but also for monitoring indeterminate lesions at risk of neoplastic transformation considering that it is cheaper and more accessible than MRI.

\section{NATURAL HISTORY OF PRENEOPLASTIC LESIONS}

Although preneoplastic lesions have been described several years ago, their natural history has been clarified only recently by prospective studies carried out on cirrhotic patients undergoing US surveillance for early detection of HCC. Early small series from Japan ${ }^{[71-73]}$ collected in a pre-dynamic imaging era, and including ultrasonically detected non malignant lesions classified according to different histologic criteria, provided preliminary evidence of the preneoplastic role of adenomatous/dysplastic nodules. More robust and convincing data derived from two successive prospective studies ${ }^{[5,74}$ comparing the natural history of different non-malignant lesions detected by ultrasounds in cirrhosis and categorized according to IWP classification. These studies confirmed that HG-DN were the true precursors of HCC with a risk of neoplastic transformation significantly higher as compared to LG-DN and LRN. Similar conclusions emerged from a single centre study on 66 LRNs and 20 DNs with a 28 -years follow-up by Sato et al. ${ }^{[75]}$. However, in these studies, the overall rate of malignant evolution of HG-DN nodules was relatively low ranging from $9 \%$ to $31 \%$ and neoplastic evolution could be documented in large regenerative/LG-DN as well albeit at a lower rate ${ }^{[5,74]}$ [Table 2]. In addition, transforming progression of HG-DNs was hardly predictable in terms of elapsing time from US detection to HCC transformation. In fact, some HG-DNs remained stable over a long time (20\% to 50\%), often exceeding the follow-up time suggested by AASLD/EASL guidelines (18 months), and some disappeared during follow-up. These data rise concern on the low PPV of morphology as a unique tool for the correct identification of nodules at risk of transformation when applied to small samples supporting a "watchful waiting" policy based on a strict radiologic surveillance. Conversely, Iavarone et al. ${ }^{[76]}$ in a retrospective-prospective study carried out on 36 non-malignant nodules histologically classified as 
Table 2. Natural history of regenerative/dysplastic nodules in cirrhosis

\begin{tabular}{|c|c|c|c|c|c|c|}
\hline Author (years) & Type & No & $\begin{array}{c}\text { Malignant } \\
\text { changes }\end{array}$ & Unchanged & Disappeared & $\begin{array}{c}\text { Time (months) to HCC } \\
\text { progression Mean (range) }\end{array}$ \\
\hline Kondo et al. ${ }^{[71]}, 2011$ & LRN & 17 & - & $13(76 \%)$ & $4(24 \%)$ & NA \\
\hline Terasaky et al. ${ }^{[72]}, 1998$ & LRN/DN & 34 & $5(15 \%)$ & $4(12 \%)$ & $25(73 \%)$ & NA \\
\hline Seki et al. ${ }^{[73]}, 2000$ & DN & 33 & $4(9 \%)$ & $14(42 \%)$ & $15(49 \%)$ & $18(16-21)$ \\
\hline Borzio et al. ${ }^{[5]}, 2003$ & LRN/DN & 90 & $28(31 \%)$ & $44(49 \%)$ & $18(20 \%)$ & $22(8-48)$ \\
\hline Kobayashi et al. ${ }^{[74]}, 2006$ & LRN/DN & 154 & $29(19 \%)$ & $81(53 \%)$ & $44(28 \%)$ & NS \\
\hline lavarone et al. ${ }^{[76]}, 2013$ & LRN/DN & 36 & $11(31 \%)$ & $21(53 \%)$ & $4(16 \%)$ & $13(7-27)$ \\
\hline Sato et al. ${ }^{[75]}, 2015$ & LRN/DN & 92 & $19(21 \%)$ & $30(32 \%)$ & $43(47 \%)$ & NS \\
\hline
\end{tabular}

NA: not available; NS: not specified. DN: dysplastic nodules; LRN: large regenerative nodules

LG- or HG-DNs found that neither size increase nor changing on enhancing pattern emerged as accurate predictors of neoplastic transformation since some DNs transformed into HCC without enlarging and others without acquiring arterial hypervascularity.

In the dynamic imaging era, the natural history of pre-malignant nodules can be extrapolated from mainly retrospective studies including nodules classified as non-enhancing/indeterminate at both CT and MRI to differentiate them from progressed HCC. Studies before 2010 and mainly based on dynamic CT, confirmed that some of these hypo-enhancing nodules carry a risk to acquire true radiologic hallmarks of malignancy over time even though this risk at an individual level still remains unpredictable. From these studies, size at baseline (larger the nodule, higher the risk) and changes in size and/or in the vascular pattern during followup (shift from hypo- to hyper-enhancing pattern on arterial phase), were reliable predictors of malignant transformation ${ }^{[77,78]}$. In the retrospective study by Chuo et al ${ }^{[79]}$ including a large series of indeterminate (hypo-vascular) small nodules observed during surveillance of HBV-related cirrhosis, emerged that old age, initial size $>1 \mathrm{~cm}$ and arterial enhancement were risk factors for neoplastic progression. The authors developed a useful and accurate risk score model for predicting HCC progression of indeterminate nodules. Unfortunately, this risk score model was not further validated in series with different etiologic liver diseases.

From 2010 onwards, most data on natural history of small hypovascular lesions observed in cirrhosis derived from studies carried out by gadoxeti-acid enhanced MRI ${ }^{[80-86]}$. In these studies neoplastic evolution was assumed to occur by acquisition of hypervascularity. Unfortunately, all these studies were retrospective and hypovascular/indeterminate nodules lacked histological classification. Furthermore, in most of these studies, one of the inclusion criteria was hypointensity at HE-phase. As previously stated, most of small nodules hypovascular, hypo-intense at He-phase, are eHCC/HG-DN. Thus, in the strict sense, these studied focused on the natural history of early hypovascular HCCs or progressed HG-DN rather than on precursor lesions as a whole ${ }^{[80-86]}$. This may partially explain why the transition rate to hyper-vascularized pHCC found in these studies was extremely high ranging from $12 \%$ to $35 \%$ at one year [Table 3].

At the best of our knowledge, only two studies by gadoexetic-acid enhanced MRI provided reliable information on the natural history of premalignant lesions with exclusion of hypovascular HCC.

The first by Kim et al. ${ }^{[85]}$ focused on hypovascular, HE hypo-intense lesions without T2W hyperintensity. Authors assumed that having excluded T2 hyperintense nodules, the risk of inclusion of eHCC among hypovascular hypointense nodules on HE phase was marginal.

In this study the rate of hypervascular transformation of precursor nodules, such as LG-DN and HG-DN was $23 \%$ at 3 years. In the second study, Sano et al.$^{[86]}$ in a large series of small hypo-vascular nodules, iso/ hyperintense at $\mathrm{HE}$ found that acquisition of hypervascularity was extremely rare (0.6\%) over 3 years followup and no nodules evolved into mature HCC after the fourth year. In this study the only independent risk factor for progression was the initial size of the nodule $(>10 \mathrm{~mm})$. In addition, the nodule growth rate showed $85 \% \mathrm{PPV}$ in predicting of hypervascularization. 
Table 3. Natural history of small hypovascular nodules at MRI and evaluated by gadoxetic acid uptake at hepatic phase

\begin{tabular}{|c|c|c|c|c|c|c|}
\hline Author (years) & Type & $\begin{array}{l}\text { HE intensity } \\
\text { at baseline }\end{array}$ & $\begin{array}{c}\text { Nodules } \\
\text { (n) }\end{array}$ & $\begin{array}{c}\text { Acquaired } \\
\text { hypervascularity }\end{array}$ & $\begin{array}{c}\text { Mean follow-up } \\
\text { (months) }\end{array}$ & Risk factors \\
\hline Kumada et al. ${ }^{[81]}, 2011$ & Retrospective & Hypo- & 49 & $6(27 \%)$ & 12 & Size $\geq 15 \mathrm{~mm}$ \\
\hline Motosugi et al. ${ }^{[80]}, 2011$ & Retrospective & Hypo- & 135 & $16(12 \%)$ & & $\begin{array}{l}\text { Size } \geq 10 \mathrm{~mm} \\
\text { Fat content } \\
\text { enlargement }\end{array}$ \\
\hline Kim et al. ${ }^{[83]}, 2012$ & Retrospective & Нypo- & 214 & $75(35 \%)$ & 11 & Hyperintensity at DWI \\
\hline Hyodo et al. ${ }^{[82]}, 2013$ & Retrospective & Нypo & 160 & $50(31 \%)$ & 12 & $\begin{array}{l}\text { Rapid growth (tumor } \\
\text { volume doubling time } \\
=542 \text { days) } \\
\text { T2W hyper-intensity }\end{array}$ \\
\hline Higaki et al. ${ }^{[84]}, 2014$ & Retrospective & Нyро & 60 & $10(17 \%)$ & 12 & Higher growth rate \\
\hline Kim et al. ${ }^{[85]}, 2016$ & Retrospective & $\begin{array}{l}\text { Hypo } \\
\text { No T2W } \\
\text { hyperintensity }\end{array}$ & 114 & $26(23 \%)$ & 42 & $\begin{array}{l}\text { T1w hyperintensity } \\
\text { Size }>10.5 \mathrm{~mm} \\
\text { Previous HCC } \\
\text { Rapid growth rate }\end{array}$ \\
\hline Sano et al. ${ }^{[86]}, 2017$ & Retrospective & Iso-hyper & 663 & $6(0.9)$ & 36 & Size $>10 \mathrm{~mm}$ \\
\hline
\end{tabular}

HCC: hepatocellular carcinoma

In summary, results from imaging-based studies provide evidence showing that indeterminate hypovascular nodules may evolve into mature HCC but this transition is hardly predictable based on initial clinical characteristics and MR imaging features. The risk and speed of neoplastic evolution of hypovascular nodules seems to depend mostly on their behaviour at HE phase on gadoexetic-acid enhanced MRI. Hypo-intense nodule at HE phase, have an elevated risk to became hypervascular pHCC in a short interval, whereas nodules iso/hyper-intense at HE phase, showed a minimal oncogenic risk ${ }^{[80-86]}$. Among the numerous risk factors found, the growth rate per se seems to have the highest positive predictive value and should be regarded as the most reliable alarm ring for radiologic evolution to progressed HCC.

\section{SURVEILLANCE AND TREATMENT}

The proper follow-up of non-malignant lesions is still debated and, theoretically, it should be dictated by the intrinsic risk of neoplastic evolution [Table 4]. Guidelines do not specifically address this issue even though a strict follow up is recommended. Once a nodule is histologically classified as dysplastic, either LG-DN or HG-DN, it should enter an enhanced follow-up the goal of which is to promptly capture its neoplastic transformation. However, indications on such an enhanced surveillance are not uniform among different guidelines. An interval of 3-4 months seems to be reasonable since it would ensure that, in case of malignant transformation, nodule would not grow beyond curability. For nodule diagnosed only by imaging, the interval should be dictated by radiologic characteristics. Hypovascularity coupled with hypo-intensity at HE by gadoexetic-acid MRI call for a strict follow up. Conversely, hypovascular nodules showing iso/ hyperintensity at HE-phase should be monitored by standard six months interval and with a follow-up no longer than 3 years. Eighteen months observation period as that recommended by AASLD/EASL guidelines seems to be inadequate since neoplastic transformation of some DN may take longer interval. As to which imaging technique should be employed, ideally it should be able to detect changes either in size and/or in vascular profile. Therefore, a dynamic imaging is preferable to standard ultrasound. CEUS, being cheaper, safer and more accessible than CT or MRI seems to be preferable in clinical practice since it can catch changes either in size or vascularity. Gadoexetate-enhancing MRI with DWI evaluation remains the recall imaging of reference to confirm neoplastic transition of nodules.

Early treatment of dysplastic nodules, which may theoretically improve survival is controversial. Unlike in others human models of gastrointestinal carcinogenesis (colo-rectal and gastroesophageal cancer) where treatment of precancerous lesions is recommended, in hepatocarcinogenetic model data supporting this policy are still lacking and evidences from the natural history of preneoplastic lesions discourage their systematic treatment. Recommendations on this issue by international guidelines are discordant. American 
Table 4. Clinical, radiologic and morphologic features useful to predict neoplastic evolution of dysplastic nodules

\begin{tabular}{ll}
\hline Features & Risk of neoplastic evolution \\
\hline Size & \\
$<1 \mathrm{~cm}$ & Low \\
$>1.5 \mathrm{~cm}$ & High \\
Arterial enhancement at CT, MRI, CEUS & \\
Hypo-vascularity & Low \\
Hyper-vascularity & High \\
HE phase at gadoxetate-enhanced MRI & \\
Iso/hyper-intense & Low \\
Hypo-intensity & High \\
DWI & \\
Hypo-intensity & Low \\
Hyper-intensity & High \\
Imaging features at follow-up & \\
Stable size & Low \\
Increasing size & High \\
Stable vascular pattern & Low \\
Acquired hyper-vascularity & High \\
Sincronous HCC & \\
No & Low \\
Yes & High \\
Histologic diagnosis & \\
LG-DN & Low \\
HG-DN & high \\
\hline & \\
\hline &
\end{tabular}

LG-DN: low-grade dysplastic nodule; HG-DN: high-grade dysplastic nodule; HCC: hepatocellular carcinoma

and European guidelines do not recommend systematic treatment of these lesions while Asian and Japanese guidelines are in favour of treatment od HG-DN. These discrepancies can be explained by the confusion in the pathological interpretation of early HCC and DNs among Japanese and Western pathologists. In particular, many of the vaguely nodular well-differentiated HCCs diagnosed by Japanese pathologists tend to be interpreted as high-grade DNs rather than HCC by Western pathologists while, many of the highgrade DNs diagnosed by Western pathologists are interpreted as well-differentiated HCC by Japanese pathologists ${ }^{[87]}$. This grey zone is particularly worrisome considering that the pursued goal is to treat any lesion arising in cirrhosis within an optimal curable stage (within $2 \mathrm{~cm}$ as the maximum diameter). However, according to western point of view, concerns are raised on the indiscriminate treatment of DNs that might be regarded as futile due to their longer and unpredictable natural history. In addition, the accurate selection of lesions with true neoplastic potential is still difficult in particular when multiple lesions are encountered. To data, only few studies addressed this issue with questionable conclusions. In 2008, Kim et al ${ }^{[88]}$ reported in a retrospective study the results of radiofrequency ablation (RFA) of 21 HG-DNs as compared to 41 small HCCs. Although complete necrosis was successfully obtained in $100 \%$ of DN, this result did not translate into either long-term overall and disease-free survival benefit owing to the occurrence of "de novo" HCCs aside the initial DNs (48\%) as the natural course of multicentric hepatocarcinogenesis. Owing to the lack and the difficulties to organize and conduct well-designed prospective controlled trials, Korean authors addressed this issue by a simulation model comparing two treatment strategies: RFA versus follow-up and timely resection. This model could not provide any evidence supporting that nodular ablation was superior to follow-up and timely resection for overall survival. Furthermore, in patients with multiple HG-DNs, RF ablation of all nodule is not clinically feasible, as it can compromise liver function ${ }^{[89]}$. In conclusion, the rationale for systematic treatment of DN in cirrhosis at present is weak and carries the risk of falling into an overtreatment, i.e., treatment of lesions which may not cause significant disease in the patient.

\section{CONCLUSION}

Like in other gastrointestinal oncogenetic models, in multistep cirrhosis-related hepatocarcinogenesis the development of HCC is preceded by sizable dysplastic lesions. The IWP classification distinguish DN into 
LG- and HG-DN, being the latter the true HCC precursors. Although relevant advances have been obtained in the last 2 decades as to morphology, and radiologic behaviour of these precursors, their management still represents a challenge for clinicians, radiologists and pathologists. In particular, major difficulties arise in surgical and transplant setting where experts have to face with these small lesions and make decisions about early treatment or simple observation without impacting negatively on the single patient outcome ${ }^{[90]}$. This remain an open and hot issue and efforts of scientific community are targeted to search and validate radiological and tissue markers helping to select lesions more prone to evolve into well-established HCC and deserving early treatment. Due to the limitations of biopsy and considering the complexity of radiologic work up, strict follow-up remains the most reliable alternative and the nodule growth the most confident and easy-to-use parameter driving decision-making process.

\section{DECLARATIONS}

\section{Authors' contributions}

Planned the review: Borzio M

Performed data research and data extraction: Borzio M, Francica G

Drafted the manuscript and approved the final version of the manuscript: Borzio M, Paladino F, Francica G Controlled for data extraction: Borzio M, Paladino F, Francica G

\section{Availability of data and materials}

Not applicable.

\section{Financial support and sponsorship}

None.

\section{Conflicts of interest}

All authors declared that there are no conflicts of interest.

\section{Ethical approval and consent to participate}

Not applicable.

\section{Consent for publication}

Not applicable.

\section{Copyright}

(C) The Author(s) 2019.

\section{REFERENCES}

1. Arakawa M, Kage M, Sugihara S, Nakashima T, Suenaga M, et al. Emergence of malignant lesions within an adenomatous hyperplastic nodule in a cirrhotic liver. Observations in five cases. Gastroenterology 1986;91:198-208.

2. Sakamoto M, Hirohashi S, Shimosato Y. Early stages of multistep hepatocarcinogenesis: adenomatous hyperplasia and early hepatocellular carcinoma. Hum Pathol 1991;22:172-8.

3. Kudo M. Multistep human hepatocarcinogenesis: correlation of imaging with pathology. J Gastroenterol 2009;44 Suppl 19:112-8.

4. International Working Party. Terminology of nodular hepatocellular lesions. Hepatology 1995;22:983-93.

5. Borzio M, Fargion S, Borzio F, Fracanzani AL, Croce AM, et al. Impact of large regenerative, low grade and high grade dysplastic nodules in hepatocellular carcinoma development. J Hepatol 2003;39:208-14.

6. Caturelli E, Solmi L, Anti M, Fusilli S, Roselli P, et al. Ultrasound-guided fine needle biopsy of early hepatocellular carcinoma complicating liver cirrhosis: a multicentre study. Gut 2004;53:1356-62.

7. Bolondi L, Gaiani S, Celli N, Golfieri R, Grigioni WF, et al. Characterization of small nodules in cirrhosis by assessment of vascularity: the problem of hypovascular hepatocellular carcinoma. Hepatology 2005;42:27-34.

8. Forner A, Vilana R, Ayuso C, Bianchi L, Solè M, et al. Diagnosis of hepatic nodules $20 \mathrm{~mm}$ or smaller in cirrhosis: prospective validation of the noninvasive diagnostic criteria for hepatocellular carcinoma. Hepatology 2008;47:97-104.

9. Sangiovanni A, Mannini MA, Iavarone M, Romeo R, Forzenigo LV, et al. The diagnostic and economic impact of contrast imaging 
techniquesin the diagnosis of small hepatocellular carcinoma in cirrhosis. Gut 2010;59:638-44.

10. ICGHN. Pathologic diagnosis of early hepatocellular carcinoma: a report of the International Consensus Group for Hepatocellular Neoplasia. Hepatology 2008;49:658-64.

11. Roncalli M, Park YN, Borzio M, Sangiovanni A, Sciarra A, et al. Premalignant and early malignant hepatocellular lesions in chronic hepatitis/cirrhosis. In: Saxena R, editor. Practical Hepatic pathology. A diagnostic approach. 2nd ed. 2018; doi: 10.1016/B978-0-32342873-6.00031-7.

12. Tateishi R, Yoshida H, Matsuyama Y, Mine N, Kondo Y, et al. Diagnostic accuracy of tumor markers for hepatocellular carcinoma: a systematic review. Hepatol Int 2008;2:17-30.

13. Di Tommaso L, Destro A, Seok JY, Balladore E, Terracciano L, et al. The application of markers (HSP70 GPC3 and GS) in liver biopsies is useful for detection of hepatocellular carcinoma. J Hepatol 2009;50:746-54.

14. Tremosini S, Forner A, Boix L, Vilana R, Bianchi L, et al. Prospective validation of an immunohistochemical panel (glypican 3, heat shock protein 70 and glutamine synthetase) in liver biopsies for diagnosis of very early hepatocellular carcinoma. Gut 2012;61:1481-7.

15. Di Tommaso L, Destro A, Fabbris V, Spagnolo G, Fracanzani AL, et al. Diagnostic accuracy of clathryn heavy chain staining in a marker panel for the diagnosis of small hepatocellular carcinoma. Hepatology 2011;53:1549-57.

16. European Association For The Study Of The Of The Liver, European Organisation For Research And Treatment Of Cancer. EASLEORTC clinical practice guidelines: management of hepatocellular carcinoma. J Hepatol 2012;56:908-43.

17. Bruix J, Sherman M, American Association for the Study of Liver Diseases. Management of hepatocellular carcinoma: an update. Hepatology 2011;53:1020-2.

18. Kudo M, Matsui O, Izumi N, Iijima H, Kadoya M, et al. JSH Consensus-based clinical practice guidelines for the management of hepatocellular carcinoma: 2014 update by the liver cancer study group of Japan. Liver Cancer 2014;3:458-68.

19. Omata M, Lii Cheng AL, Kokudo N, Kudo M, Lee JM, et al. Asia-Pacific clinical practice guidelines on the management of hepatocellular carcinoma: a 2017 update Hepatol Int 2017;11:317-70.

20. Korean Liver Cancer Study Group (KLCSG), National Cancer Center, Korea (NCC). 2014 Korean liver cancer study group-national cancer center Korea practice guideline for the management of hepatocellular carcinoma. Korean J Radiol 2015;16:465-522

21. Cartier V, Crouan B, Esvan M, Oberti F, Michalak S, et al. Suspicious liver nodule in chronic liver disease: Usefulness of a second biopsy. Diagn Interv Imaging 2018;99:493-9.

22. Leoni S, Piscaglia F, Golfieri R, Camaggi V, Vidili G, et al. The impact of vascular and nonvascular findings onthe noninvasive diagnosis of small hepatocellular carcinoma based on the EASL and AASLD criteria. Am J Gastroenterol 2010;105:599-609.

23. Khalili K, Kim TK, Jang HJ, Yazdi LK, Guindi M, et al. Indeterminate 1-2 cm nodules found on hepatocellular carcinoma surveillance: biopsy for all, some or none? Hepatology 2011;54:2048-54.

24. Durand F, Regimbeau JM, Belghiti J, Sauvanet A, Vilgrain V, et al. Assessment of the benefits and risks of percutaneousbiopsy before surgical resection of hepatocellular carcinoma. J Hepatol 2001;35:254-8.

25. Huang GT, Sheu JC, Yang PM, Lee HS, Wang TH, et al. Ultrasound-guided cutting biopsy for the diagnosis of hepato-cellular carcinoma: a study based on 420 patients. J Hepatol 1996;25:334-8.

26. Silva MA, Hegab B, Hyde C, Guo B, Buckels JA, et al. Needletrack seeding following biopsy of liver lesions in the diagnosis of hepatocellular cancer: a systematic review and meta-analysis. Gut 2008;57:1592-6.

27. Lee YJ, Lee JM, Lee JS, Lee HY, Park BH, et al. Hepatocellular carcinoma: diagnostic performance of multidetector CT and MR imaging: a systematic review and meta-analysis. Radiology 2015;275:97-109.

28. Hanna RF, Miloushev VZ, Tang A, Finklestone LA, Brejt SZ, et al. Comparative 13-year meta-analysis of the sensitivity and positive predictive value of ultrasound, CT, and MRI for detecting hepatocellular carcinoma. Abdom Radiol (NY) 2016;41:71-90.

29. Heimbach J, Kulik LM, Finn R, Sirlin CB, Abecassis MM, et al. AASLD guidelines for the treatment of hepatocellular carcinoma. Hepatology 2018;67:358-80.

30. Forner A, Llovet JM, Bruix J. Hepatocellular carcinoma. Lancet 2012;379:1245-55.

31. Sersté T, Barrau V, Ozenne V, Vullierme MP, Bedossa P, et al. Accuracy and disagreement of computed tomography and magnetic resonance imaging for the diagnosis of small hepatocellular carcinoma and dysplastic nodules: role of biopsy. Hepatology 2012;55:800-6.

32. Golfieri R, Renzulli M, Lucidi V, Corcioni B, Trevisani F, et al. Contribution of the hepatobiliary phase of Gd-EOB-DTPA-enhanced MRI to dynamic MRI in the detection of hypovascular small $(\leq 2 \mathrm{~cm})$ HCC in cirrhosis. Eur Radiol 2011;21:1233-42.

33. Ye F, Liu J, Ouyang H. Gadolinium ethoxybenzyl diethylenetriamine pentaacetic acid (Gd-EOB-DTPA)-enhanced magnetic resonance imaging and multidetector-row computed tomography for the diagnosis of hepatocellular carcinoma: a systematic review and metaanalysis. Medicine (Baltimore) 2015;94:e1157.

34. Guo J, Seo Y, Ren S, Hong S, Lee D, et al. Diagnostic performance of contrast-enhanced multidetector computed tomography and gadoxetic acid disodium-enhanced magnetic resonance imaging in detecting hepatocellular carcinoma: direct comparison and a metaanalysis. Abdom Radiol 2016;41:1960-72.

35. Kitao A, Matsui O, Yoneda N, Kozaka K, Shinmura R, et al. The uptake transporter OATP8 expression decreases during multistep hepatocarcinogenesis: correlation with gadoxetic acid enhanced MR imaging. Eur Radiol 2011;21:2056-66.

36. Kim HD, Lim YS, Han S, An J, Kim GA, et al. Evaluation of early-stage hepatocellular carcinoma by magnetic resonance imaging with gadoxetic acid detects additional lesions and increases overall survival. Gastroenterology 2015;148:1371-82.

37. Choi SH, Byun JH, Lim YS, Yu E, Lee SJ, et al. Diagnostic criteria for hepatocellular carcinoma $\leq 3 \mathrm{~cm}$ with hepatocyte-specific contrast-enhanced magnetic resonance imaging. J Hepatol 2016;64:1099-107.

38. Golfieri R, Grazioli L, Orlando E, Dormi A, Lucidi V, et al. Which is the best MRI marker of malignancy for atypical cirrhotic nodules: hypointensity in hepatobiliary phase alone or combined with other features? Classification after Gd-EOB-DTPA administration. J Magn Reson Imaging 2012;36:648-57. 
39. Ahn SS, Kim MJ, Lim JS, Hong HS, Chung YE, et al. Added value of gadoxetic acid-enhanced hepatobiliary phase MR imaging in the diagnosis of hepatocellular carcinoma. Radiology 2010;255:459-66.

40. Grazioli L, Morana G, Caudana R, Benetti A, Portolani N, et al. Hepatocellular carcinoma: correlation between gadobenate dimeglumine-enhanced MRI and pathologic findings. Invest Radiol 2000;35:25-34.

41. Gabata T, Matsui O, Kadoya M, Yoshikawa J, Ueda K, et al. Delayed MR imaging of the liver: correlation of delayed enhancement of hepatic tumors and pathologic appearance. Abdom Imaging 1998;23:309-13.

42. Manfredi R, Maresca G, Baron RL, Cotroneo AR, De Gaetano AM, et al. Delayed MR imaging of hepatocellular carcinoma enhanced by gadobenate dimeglumine (Gd-BOPTA). J Magn Reson Imaging 1999;9:704-10.

43. Narita M, Hatano E, Arizono S, Miyagawa-Hayashino A, Isoda H, et al. Expression of OATP1B3 determines uptake of Gd-EOBDTPA in hepatocellular carcinoma. J Gastroenterol 2009;44:793-8.

44. Choi JY, Lee JM, Sirlin CB. CT and MR imaging diagnosis and staging of hepatocellular carcinoma: part II. Extracellular agents, hepatobiliary agents, and ancillary imaging features. Radiology 2014;273:30-50.

45. Vilgrain V, Van Beers BE, Pastor CM. Insights into the diagnosis of hepatocellular carcinomas with hepatobiliary MRI. J Hepatol 2016;64:708-16.

46. Bartolozzi C, Battaglia V, Bargellini I, Bozzi E, Campanini D, et al. Contrast-enhanced magnetic resonance imaging of 102 nodules in cirrhosis: correlation with histological findings on explanted livers. Abdom Imaging 2013;38:290-6.

47. Kogita S, Imai Y, Okada M, Kim T, Onishi H, et al. Gd-EOB-DTPA-enhanced magnetic resonance images of hepatocellular carcinoma: correlation with histological grading and portal blood flow. Eur Radiol 2010;20:2405-13.

48. Park MJ, Kim YK, Lee MW, Lee WJ, Kim YS, et al. Small hepatocellular carcinomas: improved sensitivity by combining gadoxetic acid-enhanced and diffusion-weighted MR imaging patterns. Radiology 2012;264:761-70.

49. Kwon HJ, Byun JH, Kim JY, Hong GS, Won HJ, et al. Differentiation of small $(\leq 2 \mathrm{~cm})$ hepatocellular carcinomas from small benign nodules in cirrhotic liver on gadoxetic acidenhanced and diffusion-weighted magnetic resonance images. Abdom Imaging 2015;40:64-75.

50. Le Bihan D, Breton E, Lallemand D, Grenier P, Cabanis E, et al. MR imaging of intravoxel incoherent motions: application to diffusion and perfusion in neurologic disorders.Radiology 1986;161:401-7.

51. Li YT, Cercueil JP, Yuan J, Chen W, Loffroy R, et al. Liver intravoxel incoherent motion (IVIM) magnetic resonance imaging: a comprehensive review of published data on normal values and applications for fibrosis and tumor evaluation. Quant Imaging Med Surg 2017;7:59-78.

52. Yamada I, Aung W, Himeno Y, Nakagawa T, Shibuya H. Diffusion coefficients in abdominal organs and hepatic lesions: evaluation with intravoxel incoherent motion echo-planar MR imaging. Radiology 1999;210:617-23.

53. Iima M, Le Bihan D. Clinical intravoxel incoherent motion and diffusion MR imaging: past, present, and future. Radiology 2016;278:13-32.

54. Kwee TC, Takahara T, Koh DM, Nievelstein RA, Luijten PR. Comparison and reproducibility of ADC measurements in breathhold, respiratory triggered, and free-breathing diffusion-weighted MR imaging of the liver. J Magn Reason Imaging 2008;28:1141-8

55. Liau J, Lee J, Schroeder ME, Sirlin CB, Bydder M. Cardiac motion in diffusion weighted MRI of the liver: artifact and a method of correction. J Magn Reason Imaging 2012;35:318-27.

56. Renzulli M, Biselli B, Brocchi S, Granito A, Vasuri F, et al. New hallmark of hepatocellular carcinoma, early hepatocellular carcinoma and high-grade dysplastic nodules on Gd-EOB-DTPA MRI in patients with cirrhosis: a new diagnostic algorithm. Gut 2018;67:1674-82.

57. ACR Radiology. Available from: https://www.acr.org/Clinical-Resources/Reporting-and-Data-Systems/LIRADS/CT-MRI-LIRADS-v2017. [Last accessed on 26 Apr 2019]

58. Heimbach JK, Kulik LM, Finn RS, Sirlin CB, Abecassi MMS, et al. AASLD guidelines for the treatment of hepatocellular carcinoma. Hepatology 2018;67:358-80.

59. European Association For The Study Of The Of The Liver, European Organisation For Research And Treatment Of Cancer. EASL clinical practice guidelines: management of hepatocellular carcinoma. J Hepatol 2018;69:182-236.

60. Liu X, Jiang H, Chen J, Zhou Y, Huang Z, et al. Gadoxetic acid disodium enhanced magnetic resonance imaging outperformed multidetector computed tomography in diagnosing small hepatocellular carcinoma: a meta-analysis. Liver Transpl 2017;23:1505-18.

61. Roberts LR, Sirlin CB, Zaiem F, Almasri J, Prokop LJ, et al. Imaging for the diagnosis of hepatocellular carcinoma: a systematic review and meta-analysis. Hepatology 2018;67:401-21

62. Maruyama H, Sekimoto T, Yokosuka O. Role of contrast-enhanced ultrasonography with Sonazoid for hepatocellular carcinoma: evidence from a 10-year experience. J Gastroenterol 2016;51:421-33.

63. Takahashi M, Maruyama H, Shimada T, Kamezaki H, Sekimoto T, kanai F et al. Characterization of hepatic lesions $(<30 \mathrm{~mm})$ with liver-specific contrast agents: a comparison between ultrasound and magnetic resonance imaging. Eur J Radiol 2013;82:75-84.

64. ACR Radiology. Available from: https://www.arc.org/clinical-resources/Reporting-and-data-System/LI-RADS-v2014. [Last accessed on 26 Apr 2019]

65. Darnell A, Forner A, Rimola J, Reig M, García-Criado A, et al. Liver imaging reporting and data system with mr imaging: evaluation in nodules $20 \mathrm{~mm}$ or smaller detected in cirrhosis at screening US. Radiology:2015;275:698-707.

66. Ronot M, Fouque O, Esvan M, Lebigot J, Aube C, et al. Comparison of the accuracy of AASLD and LI-RADS criteria for the noninvasive diagnosis of HCC smaller than $3 \mathrm{~cm}$. J Hepatol 2018;68:715-23.

67. van der Pol CB, Lim CS, Sirlin CB, McGrath TA, Salameh JP, et al. Accuracy of the liver imaging reporting and data system in computed tomography and magnetic resonance image analysis of hepatocellular carcinoma or overall malignancy-a systematic review. Gastroenterology 2019;156:976-86.

68. ACR Radiology. Available from: https://www.arc.org/Clinical Resources/Reporting-and-Data-System/LI-RADSv2018. [Last accessed on 26 Apr 2019]

69. ACR Radiology. Available from: https://www.arc.org/Reporting-and-Data-System/LI-RADS/CEUS-LI-RADS-v2017 [Last accessed 
on 26 Apr 2019]

70. Terzi E, Iavarone M, Pompili M, Veronese L, Cabibbo G, et al. Contrast ultrasound LI-RADS LR-5 identifies hepatocellular carcinoma in cirrhosis in a multicenter restropective study of 1,006 nodules J Hepatol 2018;68:485-92.

71. Kondo F, Ebara M, Sugiura N, Wada K, Kita K, et al. Histological features and clinical course of large regenerative nodules: evaluation of their precancerous potentiality. Hepatology 1990;12:592-8.

72. Terasaki S, Kaneko S, Kobayashi K, Nonomura A, Nakanuma Y. Histological features predicting malignant transformation of nonmalignant hepatocellular nodules: a prospective study. Gastroenterology 1998;115:1216-22.

73. Seki S, Sakaguchi H, Kitada T, Tamori A, Takeda T, et al. Outcomes of dysplastic nodules in human cirrhotic liver: a clinicopathological study. Clin Cancer Res 2000;6:3469-73.

74. Kobayashi M, Ikeda K, Hosaka T, Sezaki H, Someya T, et al. Dysplastic nodules frequently develop into hepatocellular carcinoma in patients with chronic viral hepatitis and cirrhosis. Cancer 2006;106:636-47.

75. Sato T, Kond Fo, Ebara M, Sugiura N, Okab Se, et al, Natural history of large regenerative nodules and dysplastic nodules in liver cirrhosis: 28-year follow-up study. Hepatol Int 2015;9:330-6.

76. Iavarone M, Manini MA, Sangiovanni A, Fraquelli M, Forzenigo LV, et al. Contrast-enhanced computed tomography and ultrasoundguided liver biopsy to diagnose dysplastic liver nodules in cirrhosis. Dig Liv Dis 2013;45:43-9.

77. Takayasu K, Muramatsu Y, Mizuguchi Y, Ojima H. CT imaging of early hepatocellular carcinoma and the natural outcome of hypoattenuating nodular lesions in chronic liver disease. Oncology 2007;72 Suppl:83-91.

78. Chung JJ, Yu JS, Kim JH, Kim MJ, Kim KW. Nonhypervascular hypoattenuating nodules depicted on either portal or equilibrium phase multiphasic CT images in the cirrhotic liver. Am J Roentgenol 2008;191:207-14.

79. Chuo HJ, Kim B, Lee JD, Kang DR, Kim JK, et al. Development of risk prediction model for hepatocellular carcinoma progression of indeterminate nodules in hepatitis B virus-related cirrhotic liver. Am J Gastroenterol 2017;112:460-70.

80. Motosugi U, Ichikawa T, Sano K, Sou H, Onohara K, et al. Outcome of hypovascular hepatic nodules revealing no gadoxetic acid uptake in patients with chronic liver disease. J Magn Reson Imaging 2011;34:88-94.

81. Kumada T, Toyoda H, Tada T, Sone J, Fusijmori M, et al. Evolution of hypointense hepatocellular nodules observed only in the hepatobiliary phase ofgadoxetate disodium-enhanced MRI. AJR Am JRoentgenol 2011;197:58-63.

82. Hyodo T, Murakami T, Imai Y, Okada M, Hori M, et al. Hypovascular nodules in patients with chronic liver disease: risk factors for development of hypervascular hepatocellular carcinoma. Radiology 2013;266:480-90.

83. Kim YK, Lee WJ, Park MJ, Kim SH, Rhim H, et al. Hypovascular hypointense nodules on hepatobiliary phase gadoxetic acidenhanced MR images in patients with cirrhosis: potential of DW imaging in predicting progression to hypervascular HCC. Radiology 2012;265:104-14.

84. Higaki A, Ito K, Tamada T, Sone T, Kanki A, et al. Prognosis of small hepatocellular nodules detected only at the hepatobiliary phase of Gd-EOB-DTPA-enhanced MR imaging as hypointensity in cirrhosis or chronic hepatitis. Eur Radiol 2014;24:2476-81.

85. Kim YS, Song JS, Lee HK, Han YM. Hypovascular hypointense nodules on hepatobiliary phase without T2 hyperintensity on gadoxetic acid-enhanced MR images in patients with chronic liver disease: long-term outcomes and risk factors for hypervascular transformation. Eur Radiol 2016;26:3728-36.

86. Sano K, Ichikawa T, Motosugi U, Ichikawa S, Morisaka H, et al. Outcome of hypovascular hepatic nodules with positive uptake of gadoxetic acid in patients with cirrhosis. Eur Radiol 2017;27:518-25.

87. Kojiro M. Focus on dysplastic nodules and early hepatocellular carcinoma: an eastern point of view. Liver Transpl 2004;10:S3-8.

88. Kim SH, Lim HK, Kim MJ, Choi D, Rhim H, et al. Radiofrequency ablation of high-grade dysplastic nodules in chronic liver disease: comparison with well-differentiated hepatocellular carcinoma based on long-term results. Eur Radiol 2008;18:814-21.

89. Cho YK, Wook Chung J, Kim Y, Je Cho H, Hyun Yang S. Radiofrequency ablation of high-grade dysplastic nodules. Hepatology 2011;54:2005-11.

90. John VB, Khalili K. Not all nodules are created equal: a personalized approach to indeterminate $(<2 \mathrm{~cm})$ nodules identified on $\mathrm{HCC}$ surveillance. Am J Gastroenterol 2017;112:471-2. 